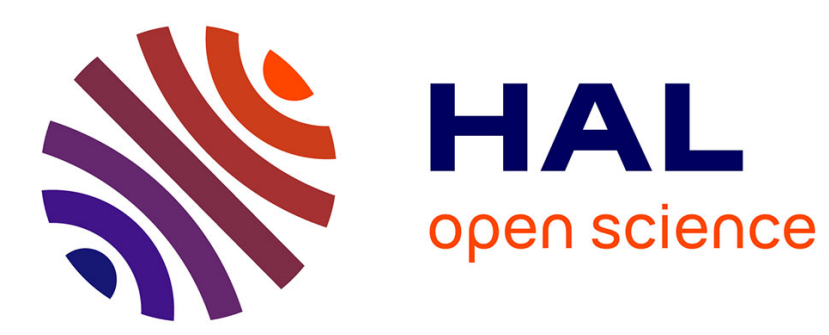

\title{
Coset partitioning for the 4-PSK space-time trellis codes
}

Pierre Viland, Gheorghe Zaharia, Jean-François Hélard

\section{To cite this version:}

Pierre Viland, Gheorghe Zaharia, Jean-François Hélard. Coset partitioning for the 4-PSK space-time trellis codes. Signals, Circuits and Systems, 2009. ISSCS 2009. International Symposium on, Jul 2009, Iasi, Romania. pp.1-4, 10.1109/ISSCS.2009.5206171 • hal-00429921

\section{HAL Id: hal-00429921 \\ https://hal.science/hal-00429921}

Submitted on 5 Nov 2009

HAL is a multi-disciplinary open access archive for the deposit and dissemination of scientific research documents, whether they are published or not. The documents may come from teaching and research institutions in France or abroad, or from public or private research centers.
L'archive ouverte pluridisciplinaire HAL, est destinée au dépôt et à la diffusion de documents scientifiques de niveau recherche, publiés ou non, émanant des établissements d'enseignement et de recherche français ou étrangers, des laboratoires publics ou privés. 


\title{
Coset Partitioning for the 4-PSK Space-Time Trellis Codes
}

\author{
Pierre Viland, Gheorghe Zaharia and Jean-Francois Hélard \\ Institute for Electronics and Telecommunications of Rennes (IETR) - UMR CNRS 6164 \\ INSA -20 avenue des Buttes de Coësmes, \\ 35043 Rennes, France \\ Email: pierre.viland@insa-rennes.fr
}

\begin{abstract}
In this paper, we present a simple method to design the best 4-PSK space-time trellis codes (STTCs) for any number of transmit antennas. This method called "coset partitioning" is based on the set partitioning proposed by Ungerboeck for the multiple input multiple output (MIMO) systems. Thanks to this method, an exhaustive search is no more required to find the best codes. Thus, new 4-PSK codes are designed and compared with good known codes. The simulation results show that these new codes outperform the existing best codes.
\end{abstract}

\section{INTRODUCTION}

The use of multiple transmit and receive antennas is a powerful solution to reduce the effect of multipath fading in wireless communications. In [1], Tarokh et al. propose the concept of space-time trellis coding to exploit the potential of MIMO systems. The STTC which combines the spatial diversity and time diversity allows to improve the error performance of the MIMO systems. In [1], the analytic bound and the first design criteria have been derived to obtain good codes. In the case of slow Rayleigh fading channels, the rank and the determinant criteria have been presented to design optimal codes. For fast Rayleigh fading channels, the product distance and the Hamming distance criteria have been proposed. In [2], Chen et al. proposed the trace criterion for slow Rayleigh fading channels. Based on the Euclidean distance, this criterion governs the performance for a great product of the number of transmit and receive antennas. However, good codes can be obtained only after the exhaustive search. Thus, the time used to search the best codes increases drastically with the number of transmit antennas.

In this paper, we propose to design the best STTCs with an adaptation of the set partitioning called "coset partitioning". The method of set partitioning has been presented by Ungerboeck in [3]-[5]. It allows to design efficiently the trellis encoder in the case of single input and single output systems. Calderbank et al. proposed an alternative to Ungerboeck's method which is simpler when the code complexity increases [6].

Ungerboeck enonciates his method with the three following rules :

1) Each constellation point has the same number of occurrences.
2) Transitions originating from the same state or merging into the same state in the trellis should be assigned subsets which contain signal points with the largest Euclidean distance.

3) Parallel paths should be assigned points of the constellation separated by the largest Euclidean distance. As there are no parallel paths in the STTCs, this rule is not important for the new design of STTCs.

The proposed method is an extension of set partitioning for the STTCs which allows to create easily the best space-time trellis codes without the exhaustive search.

The rest of the paper is organised as follows. In Section II, we present the space-time trellis encoder and the design criteria. Section III describes the method called "coset partitioning". The last section shows new 4-PSK codes obtained with the coset partitioning.

\section{SYSTEM MODEL}

\section{A. Representation of the space-time trellis encoder}

We consider a MIMO system with $n_{T}$ transmit antennas and $n_{R}$ receive antennas using a $2^{n}$-PSK modulation. For $n=2$, the 4-PSK space-time trellis encoder is presented in Fig. 1.

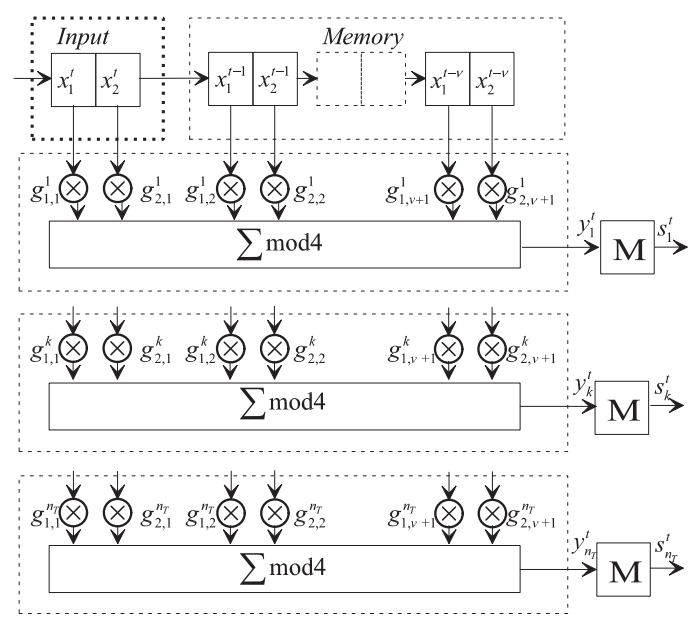

Fig. 1. Space-time trellis encoder for 4-PSK and $n_{T}$ transmit antennas

The encoder has one input block of $n$ bits and $\nu$ memory blocks of $n$ bits. The encoder state is defined by the binary 
value of the $\nu$ memory blocks of $n$ bits. At each time $t \in \mathbb{Z}$, all the bits of a block are replaced by the $n$ bits of the previous block. The $i^{t h}$ bit of the $j^{t h}$ block $x_{i}^{t-j+1}$, with $i=1 \ldots n$ and $j=1 \ldots \nu+1$, is associated to $n_{T}$ multiplier coefficients $g_{i, j}^{k} \in \mathbb{Z}_{2^{n}}, k=1 \ldots n_{T}$, where $n_{T}$ is the number of transmit antennas. With these $n_{T} \times n(\nu+1)$ coefficients, we obtain the generator matrix $G$ with $n_{T}$ lines and $\nu+1$ blocks of $n$ columns:

$$
\boldsymbol{G}=\left[G_{1}^{1} \ldots G_{n}^{1}|\ldots| G_{1}^{\nu+1} \ldots G_{n}^{\nu+1}\right]
$$

where $G_{i}^{j}=\left[g_{i, j}^{1} \ldots g_{i, j}^{n_{T}}\right]^{T} \in \mathbb{Z}_{2^{n}}^{n_{T}}$.

At each time $t$, the encoder output $\boldsymbol{y}_{x^{t}}=\left[y_{1}^{t} y_{2}^{t} \ldots y_{n_{T}}^{t}\right]^{T} \in$ $\mathbb{Z}_{2^{n}}^{n_{T}}$ is given by

$$
\boldsymbol{y}_{x^{t}}=\boldsymbol{G} \boldsymbol{x}^{t}
$$

where $\boldsymbol{x}^{t}=\left[x_{1,1}^{t} \ldots x_{1, n}^{t} . . x_{\nu+1,1}^{t} \ldots x_{\nu+1, n}^{t}\right]^{T}$ is the extendedstate at time $t$ of the $L=n(\nu+1)$ length shift register realized by the $n$ input bits followed by the $\nu$ memory blocks of $n$ bits.

In general, each encoder output $y_{k}^{t}$ is mapped onto a $2^{n}$-PSK signal given by:

$$
s_{k}^{t}=\exp \left(j \frac{\pi}{2^{n-1}} y_{k}^{t}\right)
$$

Each output signal $s_{k}^{t}$ is sent to the $k^{t h}$ transmit antenna. At each time $t$, the set of symbols transmitted simultaneously over a fading channel is given by $\boldsymbol{s}^{t}=\left[s_{1}^{t} s_{2}^{t} \ldots s_{n_{T}}^{t}\right]^{T}$.

An encoder can also be represented by a trellis, as shown in Fig. 2 for 4-PSK 4 states STTC.

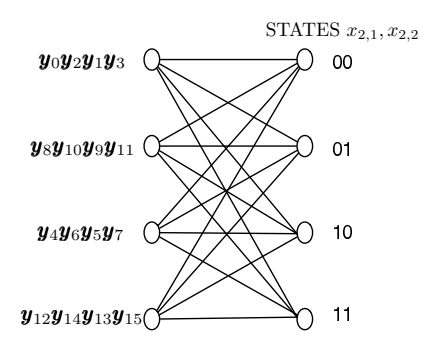

Fig. 2. 4 states 4-PSK STTC

In the trellis, the states are represented by the points and the transitions between the states by the lines. Each transition corresponds to an extended-state. The vector $\boldsymbol{y}_{i} \in \mathbb{Z}_{4}^{n_{T}}$ represents the MIMO symbol associated to an extended-state. The index $i$ is computed as the decimal value of this extendedstate with $x_{1,1}^{t}$ the least significant bit. In this example, the trellis corresponds to the generator matrix $\boldsymbol{G}=\left[y_{1} y_{2} \mid y_{4} y_{8}\right]$.

In the general case, for $2^{n}$-PSK STTCs, there are $2^{n}$ transitions originating from the same state or merging into the same state. Each MIMO symbol belongs to $\mathbb{Z}_{2^{n}}^{n_{T}}$. The number of states is given by the number of points on one column of the trellis.

\section{B. Design criteria}

The main goal of the following design criteria is to find the codes which reduce the pairwise error probability (PEP) which is the probability that the decoder selects an erroneous sequence. It is possible to represent the transmitted frame with $L_{f}$ MIMO symbols beginning at time $t=0$ by a $n_{T} \times L_{f}$ dimension matrix $\boldsymbol{S}=\left[\boldsymbol{s}^{0} \boldsymbol{s}^{1} \ldots \boldsymbol{s}^{L_{f}-1}\right]$. An error occurs if the decoder decides that another frame $\boldsymbol{E}=\left[\boldsymbol{e}^{0} \boldsymbol{e}^{1} \ldots \boldsymbol{e}^{L_{f}-1}\right]$ is transmitted. Let define the $n_{T} \times L_{f}$ difference matrix $B=E-S$ :

$$
\boldsymbol{B}=\left[\begin{array}{ccc}
e_{1}^{0}-s_{1}^{0} & \ldots & e_{1}^{L_{f}-1}-s_{1}^{L_{f}-1} \\
\vdots & \ddots & \vdots \\
e_{n_{T}}^{0}-s_{n_{T}}^{0} & \ldots & e_{n_{T}}^{L_{f}-1}-s_{n_{T}}^{L_{f}-1}
\end{array}\right]
$$

The $n_{T} \times n_{T}$ product matrix $\boldsymbol{A}=\boldsymbol{B} \boldsymbol{B}^{*}$ is introduced, where $\boldsymbol{B}^{*}$ denotes the hermitian of $\boldsymbol{B}$. We define $r=\min (\operatorname{rank}(\boldsymbol{B}))$ where $B$ is computed for all pairs of coded frames $(\boldsymbol{E}, \boldsymbol{S})$. The design criteria depend on the value of the product $r n_{R}$.

The product $r n_{R} \leq 3$ :

In this case, for a slow Rayleigh fading channel, two criteria have been proposed [1] to reduce the PEP.

- $A$ has to be a full rank matrix for any codewords $E, S$.

- For any codewords, the determinant of $A$ must be maximized.

The product $r n_{R} \geq 4$ :

In [2], it is shown that for $r n_{R} \geq 4$, the PEP is minimized if the sum of all the eigenvalues of the matrix $A$ is maximized. Since $A$ is a square matrix, the sum of all the eigenvalues is equal to the trace of the matrix $A$ :

$$
\operatorname{tr}(\boldsymbol{A})=\sum_{k=1}^{n_{T}} \lambda_{k}=\sum_{k=1}^{n_{T}}\left(\sum_{q=0}^{L_{f}-1}\left|e_{t+q}^{k}-s_{t+q}^{k}\right|^{2}\right)
$$

In other words, the trace corresponds to the Euclidean distance between the frames $\boldsymbol{E}$ and $\boldsymbol{S}$. Thus, in order to reduce the bit error rate (BER) and the frame error rate (FER), we must maximize the Euclidean distance. In this paper, we consider only this design criterion.

\section{COSET PARTITIONING FOR 4-PSK STTCS WITH $n_{T}$ TRANSMIT ANTENNAS}

\section{A. Coset decomposition of $\mathbb{Z}_{4}^{n_{T}}$}

We consider the case of a 4-PSK $2^{2 \nu}$ states STTC with $n_{T}$ transmit antennas. Each MIMO symbol belongs to the group $\mathbb{Z}_{4}^{n_{T}}$. This group can be decomposed in two sets of cosets. Each coset is defined by $C_{p}=p+C_{0}$ with $p \in \mathbb{Z}_{2}^{n_{T}}$ and $C_{0}=2 \mathbb{Z}_{2}^{n_{T}}$.

- The first set is $E_{0}=C_{0}$. This set is a normal subgroup of $\mathbb{Z}_{4}^{n_{T}}$ with the property $v=-v, \forall v \in E_{0}$.

- The other set is $E_{1}=\bigcup\left(p+C_{0}\right)$ with $p \in \mathbb{Z}_{2}^{n_{T}} \backslash[0 \cdots 0]^{T}$. Each coset $p+C_{0} \in E_{1}$ is relative to the element $v=2 p \in E_{0}$.

\section{B. Coset partitioning}

The main goal of the coset partitioning is to design easily and quickly the best STTCs thanks to the first two rules of the set partitioning. The third rule of the set partitioning is not used to design the STTCs because there are no parallel 
paths in the STTCs. The first rule of the Ungerboeck's method specifies that each point of the MIMO constellation has the same number of occurrences. In [7], [8], it has been observed that the first rule is verified by each good STTC. The STTCs verifying this first rule are called balanced codes. The coset partitioning allows to design balanced STTCs which respect also the second rule of the set partitioning: the Euclidean distance between the signals originating from the same state and merging into the same state must be maximized.

In [6], Calerbank et al. give an alternative to the set partitioning. The MIMO constellation can be seen as a finite subgroup of $\mathbb{Z}_{2^{n}}^{n_{T}}$. This group is partitioned in cosets. The goal of this method is that all the transitions originating from or merging into the same state belong to the same coset. The elements of these cosets must be separated by the largest Euclidean distances.

For a 4-PSK $2^{2 \nu}$ states STTC, the generator matrix has $\nu+1$ blocks of 2 columns. To design the STTCs with the coset partitioning, we propose that the 2 columns of each block can generate a subgroup of $\mathbb{Z}_{4}^{n_{T}}$. Therefore, for each block $k$, the following rule must be respected:

- The first column $G_{1}^{k}$ must belong to $C_{0}$ with $1 \leq k \leq$ $\nu+1$.

- The column $G_{2}^{k}$ with $1 \leq k \leq \nu+1$ must belong to the coset relative to the first column $G_{1}^{k}$ or to $C_{0}$.

After these choices, it is possible to permute the columns and the lines within each block to obtain codes which respect the coset partitioning. Further on, we search the codes with the best trace.

\section{Example of a 4-PSK 16 states STTC with $n_{T}$ transmit antennas}

In the case of a 4-PSK 16 states STTC with $n_{T}$ transmit antennas, the matrix $G$ has 3 blocks of 2 columns: $B_{1}=$ $\left[G_{1}^{1}, G_{2}^{1}\right], B_{2}=\left[G_{1}^{2}, G_{2}^{2}\right]$ and $B_{3}=\left[G_{1}^{3}, G_{2}^{3}\right]$ which give $\boldsymbol{G}=$ $\left[G_{1}^{1} G_{2}^{1}\left|G_{1}^{2} G_{2}^{2}\right| G_{1}^{3} G_{2}^{3}\right]$. Each column belongs to the group $\mathbb{Z}_{4}^{n_{T}}$.

The first step to design the code with the coset partitioning is to select the cosets originating from the same state and merging into the same state, i.e. the elements of the blocks $B_{1}$ and $B_{3}$. These blocks of the generator matrix must generate respectively the subgroups $\Lambda_{1}^{F}=\left\{\boldsymbol{y}_{0}, \boldsymbol{y}_{1}, \boldsymbol{y}_{2}, \boldsymbol{y}_{3}=\boldsymbol{y}_{1}+\right.$ $\left.\boldsymbol{y}_{2} \bmod 4\right\}$ and $\Lambda_{1}^{M}=\left\{\boldsymbol{y}_{0}, \boldsymbol{y}_{16}, \boldsymbol{y}_{32}, \boldsymbol{y}_{48}=\boldsymbol{y}_{16}+\boldsymbol{y}_{32} \bmod 4\right\}$. The set $\Lambda_{\nu+1}$ of generated MIMO symbols can be decomposed into two sets of cosets $\Lambda_{\nu+1} / \Lambda_{1}^{F}$ and $\Lambda_{\nu+1} / \Lambda_{1}^{M}$ where each coset corresponds to the set of transitions originating from the same state and the transitions merging into the same state respectively. The trellis given in Fig. 3 corresponds to a 4-PSK 16 states STTC designed with the coset partitioning.

The second step is the selection of the columns $G_{1}^{2}$ and $G_{2}^{2}$. These vectors must also generate a subgroup. Thus, it is possible to represent a simplified trellis, as shown in Fig. 4.

In this case, the points $x_{3,1} x_{3,2} \in\{00,01,10,11\}$ of Fig. 4 are defined as the 'states of level 2'. Each state of level $2 x_{3,1} x_{3,2}$ is constituted by 4 real states $\bigcup\left(x_{2,1} x_{2,2} x_{3,1} x_{3,2}\right) \backslash\left\{x_{2,1}, x_{2,2}\right\} \in\{0,1\}$. The new lines correspond to the 'transitions of level 2' which are constituted

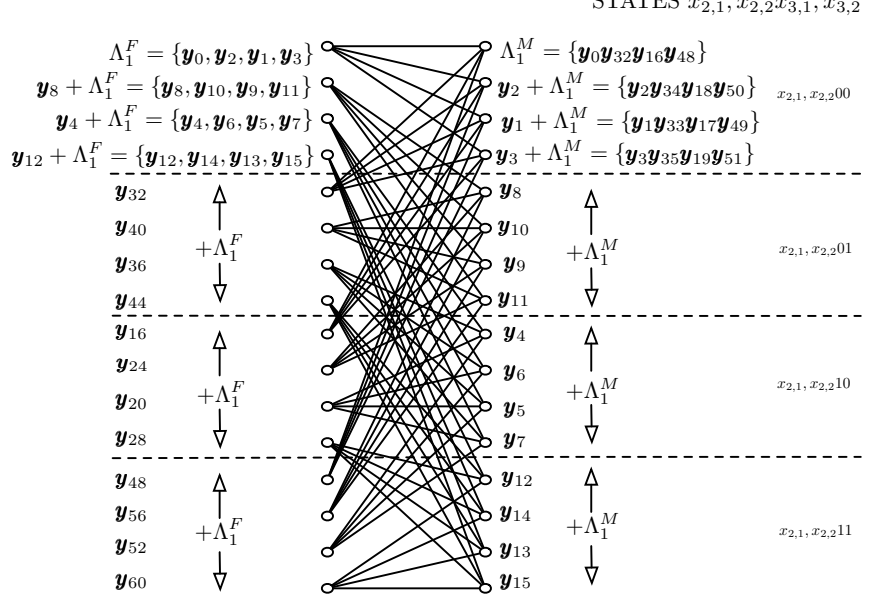

Fig. 3. 16 states 4-PSK STTC with cosets

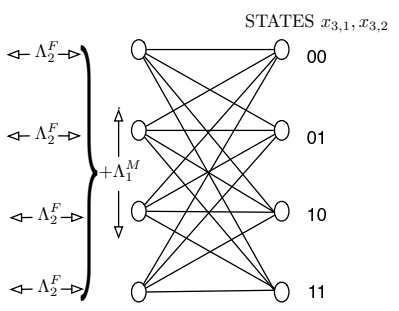

Fig. 4. Simplified representation of the trellis

by 4 real transitions. If a coset partitioning is used, then $\Lambda_{2}^{F}=\Lambda_{1}^{F} \cup\left(\Lambda_{1}^{F}+\boldsymbol{y}_{4}\right) \cup\left(\Lambda_{1}^{F}+\boldsymbol{y}_{8}\right) \cup\left(\Lambda_{1}^{F}+\boldsymbol{y}_{12}\right)$ is a subgroup of $\mathbb{Z}_{2^{n}}^{n_{T}}$ (with $\boldsymbol{y}_{4}+\boldsymbol{y}_{8}=\boldsymbol{y}_{12} \bmod 4$ ). Thus, a partition chain is obtained:

$$
\Lambda_{\nu+1} / \Lambda_{2}^{F} / \Lambda_{1}^{F}
$$

The Euclidean distances between the elements of $\Lambda_{2}^{F}$ must be maximized to obtain good codes thanks to the choice of $\boldsymbol{y}_{4}$ and $\boldsymbol{y}_{8}$. We can also make a chain partition with the MIMO symbols merging into the different levels of states:

$$
\Lambda_{\nu+1} / \Lambda_{2}^{M} / \Lambda_{1}^{M}
$$

Each element of $\Lambda_{\nu+1} / \Lambda_{2}^{M}$ corresponds to one coset of MIMO symbols merging into a same state of level 2. As shown in Fig. $4, \Lambda_{2}^{M}$ is given by: $\Lambda_{2}^{M}=\Lambda_{1}^{M}+\Lambda_{1}^{F}$. Thus, $\Lambda_{2}^{M}$ is already defined by the previous selections.

Finally, we permute the columns of each block to find the codes with the best trace.

Remark: Some codes have their first $i$ null columns in the $(\nu+1)^{t h}$ block with $0<i<n$. The number of states is $2^{n \nu-i}$. In this case, the columns which generate the subgroup $\Lambda_{1}^{M}$ are the $i$ first columns of the $\nu^{t h}$ block and the last $n-i$ columns of the $(\nu+1)^{t h}$ block.

\section{NEW CODES AND THEIR PERFORMANCE}

Using the proposed design method, new 4-PSK codes are obtained for 3 and 4 transmit antennas. Table I shows these 
new codes for 32/64 states with 3 transmit antennas and 4/8/64 states with 4 transmit antennas. Their minimum traces are compared to the traces of corresponding Chen's codes proposed in [9]. For each new code, the Euclidean distances between the MIMO symbols merging into or originating from the same state respectively are better than the corresponding Chen's codes.

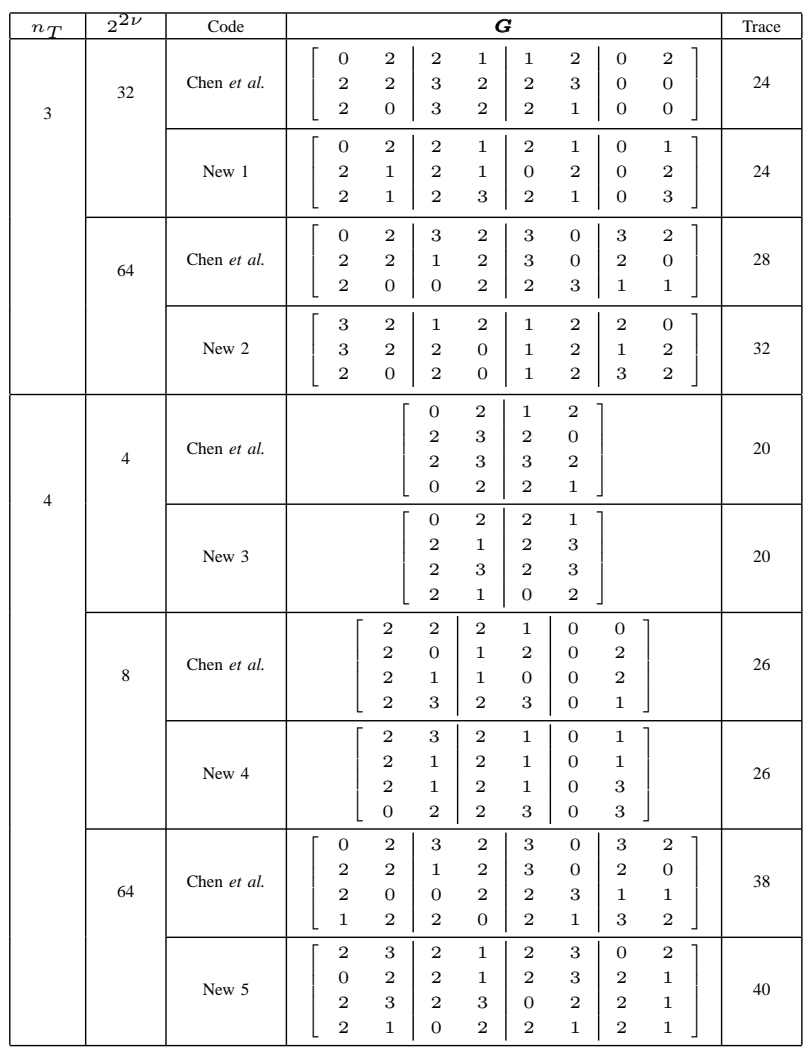

TABLE I

NEW 4-PSK CODES BASED ON THE EUCLIDEAN DISTANCE CRITERIA

For the simulation, two receive antennas are considered. The channel is a slow Rayleigh fading channel and each frame has 130 MIMO symbols. The channel coefficients are assumed to be known at the decoder. The decoding is performed by the Viterbi's algorithm. We observe the performance of a 4-PSK 32 states STTC with 3 transmit antennas and 64 states STTCs with 3/4 transmit antennas in Fig. 5 and 4-PSK 4/8 states STTCs with 4 transmit antennas in Fig. 6. Each new code outperforms slightly the corresponding Chen's code.

\section{CONCLUSiON}

In this paper, an efficient method to design the best 4-PSK STTCs with $n_{T}$ transmit anntennas has been presented. This new method is based on the set partitioning proposed by Ungerboeck and an alternative of this work proposed by Calderbank. Based on this new design method, new 4-PSK STTCs with 3 and 4 transmit antennas have been proposed.

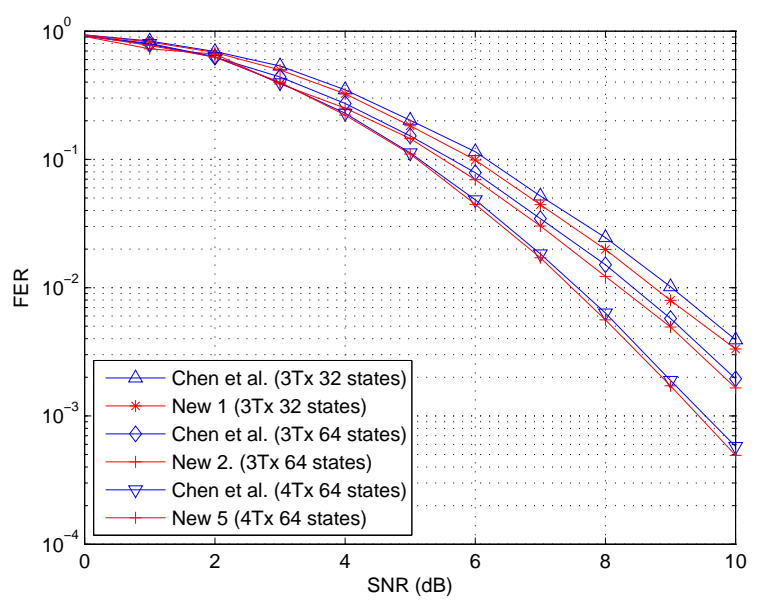

Fig. 5. Performance of 4-PSK STTCs with $3 / 4$ transmit antennas

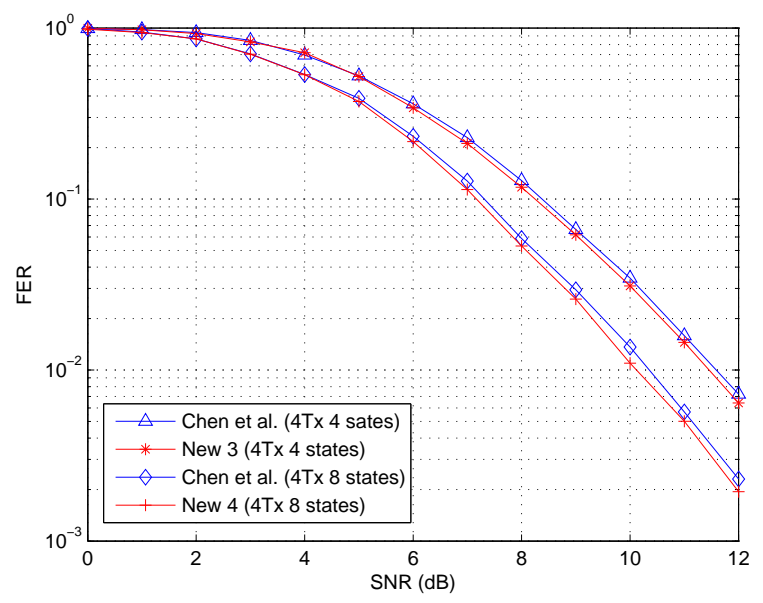

Fig. 6. Performance of 4-PSK 4/8 states STTCs with 4 transmit antennas

\section{REFERENCES}

[1] V. Tarokh, N. Seshadri, and A. Calderbank, "Space-time codes for high data rate wireless communication: Performance criterion and code construction," IEEE Trans. Inf. Theory, vol. 44, no. 2, pp. 744-765, Mar. 1998.

[2] Z. Chen, J. Yuan, and B. Vucetic, "Improved space-time trellis coded modulation scheme on slow fading channels," Electron. Lett., vol. 37, no. 7, pp. 440-441, Mar. 2001.

[3] G. Ungerboeck, "Channel coding with multilevel/phase signals," IEEE Trans. Inf. Theory, vol. 28, pp. 55-67, Jan. 1982.

[4] _ , "Trellis-coded modulation with redundant signal sets part I: Introduction," Comm. Magazine, IEEE, vol. 25, pp. 5-11, Feb. 1987.

[5] - "Trellis-coded modulation with redundant signal sets part II: State of the art," Comm. Magazine, IEEE, vol. 25, pp. 12-21, Feb. 1987.

[6] A. Calderbank and N. Sloane, "New trellis codes based on lattices and cosets," IEEE Trans. Inf. Theory, vol. 33, pp. 177-195, Mar. 1987.

[7] T. M. H. Ngo, G. Zaharia, S. Bougeard, and J.-F. Helard, "4-PSK Balanced STTC with Two Transmit Antennas," in Proc. IEEE VTC 2007Spring, Apr. 2007, pp. 1693-1697.

[8] P. Viland, G. Zaharia, and J.-F. Helard, "A new method to design balanced space-time trellis codes for several transmit antennas," in Proc. IEEE GLOBECOM 2008, Dec. 2008, pp. 1-5.

[9] Z. Chen, B. Vucetic, J. Yuan, and K. L. Lo, "Space-time trellis codes with two, three and four transmit antennas in quasi-static flat fading channels," in Proc. IEEE ICC 2002, vol. 3, May 2002, pp. 1589-1595. 